\title{
Artículo de Revisión \\ Demodicosis: Revisión de la literatura
}

\author{
Juan Andreani ${ }^{1}$, Fernando Pérez², María Teresa Molina ${ }^{3,4}$, Raúl de la Fuente ${ }^{5 .}$
}

\section{RESUMen}

La demodicosis es una patología cutánea crónica caracterizada por lesiones eritemato-maculares pruriginosas, cuyo agente causal son ácaros foliculares del género Demodex. Presenta un abanico amplio y polimorfo de manifestaciones clínicas, donde la sospecha clínica se presentará frente a una erupción facial crónica persistente o recurrente, resistente a terapia convencional y de distribución asimétrica. El diagnóstico definitivo es dificil, y requiere un cuadro clínico compatible y la presencia de alta densidad de Demodex.

El siguiente documento hace una revisión de conceptos con respecto a la patogenia, clínica, diagnóstico y tratamiento de esta entidad.

Palabras claves: Ácaros; Folículo piloso; Glándulas sebáceas; Enfermedades cutáneas.

\section{SUMMARY}

Demodicosis is a chronic skin condition characterized by itchy erythematous macular lesions whose causal agents are gender follicular Demodex mites. This entity presents a wide and polymorphous range of clinical manifestations, in which clinical suspicion appears in case of persistent or recurrent chronic facial rash, resistant to conventional therapy and with an asymmetric distribution. The definitive diagnosis is hard to reach, and requires a compatible clinical picture and a high density of Demodex.

The following document is a review of concepts regarding pathogenesis, symptoms, diagnosis and treatment of this disease.

Key words: Acari; Hair follicle; Sebaceous glands; Skin diseases.
L a demodicosis es una enfermedad cutánea crónica caracterizada por lesiones eritemato-maculares pruriginosas, que afecta principalmente la zona facial, cuyo agente causal son ácaros foliculares del género Demodex. Este término es más correcto que el utilizado anteriormente, demodicidosis, pues el sufijo "id" implica un estado de hipersensibilidad a productos del patógeno, mecanismo no involucrado en la teoría actual de la patogenia de la enfermedad. ${ }^{1}$ Solo dos especies de Demodex (D. folliculorum y D. brevis) se han identificado en humanos. Son flora comensal común de la unidad pilosebácea, ${ }^{2}$ pero también han sido implicados como agentes causales de Pitiriasis folliculorum, Rosacea-like demodicosis, rosácea papulopustular, demodicosis gravis y blefaritis. ${ }^{3}$

${ }^{1}$ Servicio de Dermatología, Hospital del Salvador, Santiago. ${ }^{2}$ Dermatólogo, práctica en consulta privada. ${ }^{3}$ Servicio de Dermatología, Hospital Clínico Universidad de Chile, Santiago. ${ }^{4}$ Departamento de Dermatología, Facultad de Medicina, Universidad de Chile, Santiago. ${ }^{5}$ Facultad de Medicina, Universidad de Chile, Santiago.

Correspondencia: Raúl de la Fuente, Facultad de Medicina Universidad de Chile. Correo electrónico: rauldelaes@yahoo.es. Teléfonos: +56 2 25270381, +56 9 91874236. Dirección: Independencia 1027, Independencia, Santiago, Chile. Código Postal: 8380000.

\section{Patogenia}

Demodex spp. pertenece a la familia Demodicidae, de la clase Arachnidia, en el orden Acarina Los ácaros del género Demodex, de 0,3 mm de longitud promedio, se adquieren poco después del nacimiento y se consideran flora normal de la unidad pilosebácea en humanos, ubicándose principalmente en la cara, cuero cabelludo y región superior del tronco. D. folliculorum se encuentra habitualmente en el infundíbulo folicular y D. brevis, en los ductos sebáceos y las glándulas tarsales. ${ }^{4}$

Crecen en número en proporción a la disposición de alimento (secreción sebácea), por lo que aumentan en forma importante en la pubertad, cuando proliferan las glándulas sebáceas. Por esto, se concentran preferentemente en zonas con producción activa de secreción sebácea, como la cara (nariz, mejillas, frente, sien, mentón) y conducto auditivo externo. ${ }^{2}$ También se encuentran en cuero cabelludo, cuello y tronco.

D. folliculorum y D. brevis se encontraron en aproximadamente el 10\% de las biopsias de piel sana. Ambas especies se encuentran más comúnmente en la cara. D. folliculorum es el ácaro predominante, pero D. brevis tiene una distribución más amplia en el cuerpo ${ }^{3}$. Su pre- 
valencia va aumentando con la edad, con una tasa de colonización normal de entre $20-100 \%$ en adultos. ${ }^{5}$

En animales está bien establecida la relación causal entre Demodex y sarna, pero en humanos el potencial patogénico de este agente es controvertido, debido a que es ubicuo y la colonización frecuentemente no se asocia a síntomas.

Son varios los mecanismos patogénicos propuestos para esta enfermedad, incluyendo: bloqueo de los folículos y ductos sebáceos por los ácaros o por hiperqueratosis reactiva, estimulación de la respuesta inmune humoral y celular por los ácaros y sus productos de desecho, una reacción granulomatosa a cuerpo extraño hacia el esqueleto de quitina del ácaro y un rol como vector para infección bacteriana. ${ }^{2}$

Se cree que los ácaros Demodex juegan un rol patogénico cuando se encuentran penetrando la dermis, ${ }^{6,7}$ cuando se presentan en número excesivo (operacionalmente definido por densidad mayor a 5 ácaros en un folículo piloso o en $1 \mathrm{~cm}^{2}$ ), cuando hay sobreinfección bacteriana o el huésped presenta una inmunodeficiencia primaria o secundaria.

La variación en las manifestaciones clínicas y la histopatología de la demodicosis pueden depender del grado de infestación por Demodex, la duración de la enfermedad, la edad y estado de salud general del huésped, y del estadio evolutivo de las lesiones individuales.

Este ácaro ha sido implicado en la patogenia de rosácea papulopustular, pitiriasis folliculorum, demodicosis rosácea-like, demodicosis gravis (demodicosis rosácea granulomatosa-like) y blefaritis., ${ }^{2,3}$

\section{Respuesta inmune a Demodex}

Los humanos comúnmente montan una respuesta vigorosa a los ácaros de Demodex, aunque esta respuesta varía dependiendo del tipo de HLA. Se ha establecido asociación entre demodicosis y los haplotipos HLA Cw2 y Cw44, esto asociado a una disminución en el número de células natural killer; mientras que individuos con fenotipo HLA A2 son 2,9 veces más resistentes a la demodicosis $^{4}$. Así, los individuos que carecen del fenotipo HLA A2 presentan con mayor frecuencia lesiones papulares profundas y papulopustulares. ${ }^{8}$ En pacientes portadores de VIH, el ácaro puede producir erupción pruriginosa que involucra las zonas facial, preesternal e interescapular, siendo más frecuentes en individuos en etapa SIDA clínica y con un recuento de CD4 menor a $200 / \mathrm{mm}^{3}{ }^{9}{ }^{9}$ También se ha encontrado alta densidad de Demodex en pacientes con linfoma o leucemia, especialmente leucemia mielocítica, que clínicamente se ha manifestado con erupción facial. ${ }^{10}$

En inmunocompetentes, solo un pequeño número desarrolla la enfermedad. En ellos se ha observado una tendencia de los linfocitos a la apoptosis aumentada, en forma paralela al aumento de la densidad de ácaros, lo que resultaría en una inmunosupresión local causada por este agente, que les permitiría sobrevivir en la piel del huésped. ${ }^{11}$

\section{Clínica}

La demodicosis presenta un abanico amplio y polimorfo de manifestaciones clínicas, donde se ha sugerido como criterios de sospecha de la enfermedad la presencia de una erupción facial crónica persistente o recurrente, resistente a terapia convencional y de distribución asimétrica.

La primera clasificación, surgió a partir de Ayres, ${ }^{1}$ donde se describió dos formas clínicas de infección por Demodex folliculorum en los seres humanos:

- la pitiriasis folliculorum

- la demodicidosis rosácea símil.

En el año 2003, se propuso agregar como dentro de las dermatosis que pueden ser colonizadas e infectadas por demodex: ${ }^{12}$

- Rosácea esteroidal: cuadro caracterizado por un eritema intenso, descamativo, cubierto por papulopústulas redondas foliculares, ubicadas en zona centro facial, perioral y periocular. Se ha descrito asociación a proliferación de Demodex.

- Dermatitis rosaceiforme inducida por tacrolimus tópico (0.1-0.3\%): inmunosupresor usado para el manejo de varias enfermedades de la piel con lesiones predominantemente inflamatorias. Se ha descrito casos de pacientes que desarrollan lesiones granulomatosas rosácea símil durante el tratamiento. La patogenia de esta condición se podría explicar por las propiedades inmunosupresoras del tacrolimus, asociado a la propiedad oclusiva 
del ungüento, que pudieran facilitar el sobrecrecimiento en los folículos de D. follicullorum en pacientes susceptibles; además por las propiedades vasoactivas de la droga se favorecería el desarrollo de flushing.

La clasificación propuesta el año $2009,{ }^{4}$ se basa en las formas cínicas y morfológicas de las lesiones, donde por orden de frecuencia se observaron:

1. Erupción eritematosa-papulopustular rosácea-símil: corresponde a la manifestación más frecuente, caracterizada por la presencia de eritema con descamación folicular y múltiples papulopústulas pequeñas, de inicio súbito, progresión rápida, con prurito y ardor. No hay historia de fotosensibilidad, eritema persistente ni flushing y al examen físico las lesiones son de distribución asimétrica, sin presencia de telangiectasias significativas. ${ }^{2}$

2. Erupción papulopustular perioral o párpados (dermatitis perioral-símil): caracterizadas por eritema y papulopústulas múltiples de ubicación perioral, que a la histología presenta infiltrado linfohistiocítico con neutrófilos perifolicular, con Demodex mayor a $5 \mathrm{D} / \mathrm{cm}^{2}$ en el infiltrado infundibular. ${ }^{4}$

3. Demodicosis gravis (Rosácea granulomatosa-símil): presenta características clínicas similares a la rosácea granulomatosa severa y se caracteriza patológicamente por granulomas dérmicos con caseificación central y restos de ácaros fagocitados por células gigantes. ${ }^{4}$

4. Pitiriasis folliculorum: se presenta como eritema facial tenue, de acentuación folicular, con escamas foliculares muy pequeñas, finas, blanquecinas y secas, formando un tapón folicular, de textura rugosa (como papel de lija). ${ }^{3}$ Esta forma afecta más frecuentemente a mujeres, con historia de aplicación de maquillajes densos o lavado infrecuente de cara.

Otras clasificaciones descritas son las:

- Foliculitis por Demodex, que consiste en pústulas foliculares, sin manifestaciones de pitiriasis folliculorum (escamas y eritema), ${ }^{3}$ de distribución unilateral.

- Abscesos por Demodex, que se presenta como papulopústulas y nódulos profundos, de localización unilateral, que generalmente son resistentes a tratamientos previos con antibióticos, isotretinoína y acaricidas. ${ }^{13}$

- Blefaritis por Demodex, que es una foliculitis de las pestañas, cuyos síntomas principales son prurito, sensación de ardor y cuerpo extraño, eritema del borde palpebral y visión borrosa. Dentro de los síntomas se incluye caspa cilíndrica, alteraciones de las pestañas, inflamación del margen palpebral, disfunción de las glándulas tarsales, blefaroconjuntivitis y blefaroqueratitis. Muchos pacientes cursan con Dermatitis seborreica con compromiso de párpados, otros tienen blefaritis asociada con rosácea. ${ }^{14}$ El diagnóstico se realiza por la visualización de folículos llenos de ácaros.

\section{Demodex, Pitiriasis Folligulorum y Rosácea Papulo Pustular (RPP)}

La pitiriasis folliculorum y la RPP según algunos autores, estarían interrelacionadas entre sí: es así como en piel sana, sin alteración en inmunidad innata, la catelicidina estaría en niveles normales. Las catecilidinas son péptidos antimicrobianos encontrados en mamíferos, aves y peces, que se presentan en diversas enfermedades inflamatorias de la piel, como dermatitis atópica, psoriasis, acné y rosácea, y constituyen una primera línea de defensa frente a infecciones cutáneas. En el ser humano se ha identificado solo un gen para catelicidina (CAMP), cuyo péptido activo, LL-37, es el único que se expresa en nuestra especie. ${ }^{15}$ En condiciones normales, la acción de LL-37 seria predominante y la inmunidad controlaría la proliferación del Demodex, en cambio, de acuerdo a esta hipótesis fisiopatológica propuesta, durante una primera etapa, habría un defecto específico de la inmunidad tanto innata como adquirida contra el Demodex que permitiría su proliferación, lo que clínicamente se expresaría como Pitiriasis Foliculorum, en la cual, a pesar de haber una alta densidad de demódex, este no genera una reacción inflamatoria evidenciable clínicamente. La inmunidad mediada por células es leve o está ausente probablemente debido a un defecto genético específico de la inmunidad innata y agravada por una inmunodepresión local inducida por el Demodex. En una segunda etapa, después de meses o años, cuando el daño del epitelio es significativo y el Demodex penetra en la dermis estimulando al sistema inmune, se produce una respuesta exagerada y no totalmente efectiva induciendo la aparición de pápulas y pústulas, lo que se expresaría como RPP, la reacción inmune es exagerada con aumento de los TLR2, excesiva producción de la enzima tríptica del estrato corneo, también conocida como calicreína 5 (KLK5), y de la catelicidina LL-37, lo que desencadena una reacción de hipersensibilidad tipo IV, con la consiguiente formación de pápulas y pústulas. La 
enzima antes mencionada es una proteasa que divide al péptido inactivo CAP18 (cationic antimicrobial protein), generándose el péptido activo LL-37, entre cuyas funciones se cuentan: quimiotaxis de leucocitos, angiogénesis y expresión de componentes de la matriz extracelular. Ya que estos efectos son similares a los cambios observados en la rosácea, se considera actualmente que la expresión anormal de catelicidina es un factor importante en la patogenia. Esto se ha verificado al determinarse experimentalmente que los niveles tanto de KLK5 como de LL-37 son mucho mayores en la piel de sujetos con rosácea que en la de aquellos que no presentan la enfermedad. Asimismo, en ratones sometidos a deleción del gen CAMP, se ha observado una mucho menor respuesta inflamatoria a estímulos irritantes, los que dan cuenta de las manifestaciones clínicas de la rosácea. ${ }^{16}$ Esta reacción inmune limita la proliferación del Demodex, pero solo parcialmente. Cuando el Demodex penetra en la dermis se genera una reacción granulomatosa. ${ }^{17}$

\section{Diagnóstico}

El diagnóstico de demodicosis es complicado debido a que los ácaros también se encuentran en la piel de población sana. El diagnóstico definitivo requiere un cuadro clínico compatible y la presencia de alta densidad de Demodex (Dd). Esta puede estudiarse mediante examen microscópico directo con $\mathrm{KOH}$ de secreción de glándulas sebáceas o Biopsia cutánea superficial estandarizada con cianoacrilato (SSSB), considerando patogénica una Dd mayor a 5 ácaros por folículo o por $\mathrm{cm}^{2}$, respectivamente. Actualmente la técnica recomendada es esta última, por presentar una aparente mayor sensibilidad. Otra herramienta diagnóstica es la biopsia cutánea (HE), que permite distinguir entre las formas clínicas de la condición: pitiriasis folliculorum (hiperqueratosis folicular rellena por ácaros, con infiltrado linfocitario perivascular y dérmico difuso), demodicosis rosácea-like (presencia del ácaro en el infundíbulo folicular asociado a infiltrado mononuclear perifolicular) y demodicosis gravis (con granulomas dérmicos con necrosis caseificante central, restos de ácaros fagocitados y células gigantes tipo Langhans). Otro criterio diagnóstico que se ha propuesto es la respuesta a la terapia anti-Demodex. La dermatoscopía también es útil para diagnosticar la demodicosis: un dermatoscopio de luz polarizada puede observar la cola del Demodex en las aperturas foliculares y las aperturas foliculares dilatadas. Además, se observa- rían vasos sanguíneos reticulares dilatados posicionados horizontalmente. $^{18}$

\section{Tratamiento}

Numerosas opciones terapéuticas se han utilizado en las enfermedades de la piel asociadas a Demodex, tanto tratamientos tópicos, como por vía oral. Se ha reportado eficacia terapéutica de ácido salicílico, sulfuro sublima$\mathrm{do}^{7}$, metronidazol, crotamitón, ivermectina oral junto con permetrina tópica y retinoides orales o tópicos. ${ }^{19}$ Dada la imposibilidad de cultivar estos ácaros, resulta difícil estudiar el posible efecto a largo plazo de los fármacos sobre la viabilidad de Demodex. Hasta la fecha no existe un estándar de tratamiento, hecho que pudiera explicarse por el gran espectro de manifestaciones clínicas de la enfermedad. En aquellos casos de demodicosis moderada, agentes tópicos que promueven descamación (como ácido salicílico y retinoides) por sí solos podrían ser efectivos gracias a que previenen la oclusión de los folículos y aceleran la muda de la epidermis, permitiendo así la eliminación de los ácaros y de sus productos de desecho. Por otra parte, se plantea que la efectividad de los antibióticos orales en el tratamiento estaría dada por una inhibición la activación de KLK5, la que, al igual que las catelicidinas, presentan un aumento de sus niveles en demodicosis y otras entidades como la rosácea. Esto, debido a una inhibición de las metaloproteinasas de matriz en los queratinocitos. ${ }^{20}$

En la literatura encontramos numerosos trabajos que señalan la efectividad del tratamiento con metronidazol tópico (gel 0.75-1 \%) y/u oral (250 mg 3 veces/día o 500 mg 2veces/día por 1-3 semanas), ${ }^{4,13,21}$ mientras otros han reportado respuesta al tratamiento tópico, pero sin resolución de la enfermedad; ${ }^{22}$ supresión de la enfermedad pero sin disminución de la población de Demodex ${ }^{18} \mathrm{e}$ inefectividad absoluta. En casos en que no se logró buena respuesta se usó crotamiton 10\% tópico, que elimina la población de Demodex y es curativo. El mecanismo de la respuesta terapéutica a metronidazol es desconocido, no pudiendo atribuirse a actividad antiparasitaria contra Demodex, porque in vitro el ácaro sobrevive a concentraciones de metronidazol de hasta $1 \mathrm{mg} / \mathrm{dl}$, la que no puede alcanzarse in vivo. Se ha propuesto la posibilidad de que algún metabolito de metronidazol actúe como agente acaricida, así como también una posible actividad anti-inflamatoria, por influencia sobre linfocitos $\mathrm{T}$ y moléculas de adhesión y la alteración de la función de los 
neutrófilos y el efecto antioxidante del metronizadol. ${ }^{23}$

Se ha señalado también efectividad del tratamiento con permetrina 5\% tópica, sola o asociada a ivermetina sistémica (200 ug/kg peso), ${ }^{11}$ incluyendo un caso de demodicosis nódulo-abscedante refractario a metronidazol endovenoso. ${ }^{24}$

Existe escasa evidencia que compare la efectividad de los agentes terapéuticos mencionados. Entre los tratamientos tópicos, un ensayo clínico randomizado que incluyó 30 pacientes, comparó la efectividad de metronidazol $2 \%$, permetrina $1 \%$, sulfuro sublimado $10 \%$, lindano $1 \%$, crotamitón $10 \%$ y benzoato de benzilo $10 \%$, demostrándose actividad acaricida de este último (a costa de un efecto irritante) y de crotamitón. Pudiera ser que, en este trabajo, permetrina no haya presentado efecto acaricida significativo debido a la concentración utilizada. ${ }^{11}$

La ivermectina 1\% tópica utilizada tanto para Demodicosis como para RPP, posee propiedades anti-inflamatorias y anti-parasitarias. Después de 12 semanas de tratamiento, los sujetos tratados con crema de ivermectina $1 \%$ tenían una reducción significativamente mayor en los síntomas y una mejoría importante en la calidad de vida al compararlos con los sujetos que recibieron vehículo placebo. Además, los síntomas continuaron mejorando con el tratamiento sistémico prolongado (40 semanas). ${ }^{25}$

\section{Conclusiones}

Los ácaros del género Demodex son considerados como flora comensal de la piel en humanos, sin embargo, están asociados a patologías dermatológicas, siendo frecuentemente subdiagnosticado como agente causal debido al polimorfismo de sus manifestaciones clínicas, el desconocimiento de la entidad por parte de los dermatólogos y la dificultad en el diagnóstico diferencial con otros cuadros. La demodicosis debe considerarse dentro del diagnóstico diferencial de dermatosis faciales, recurrentes, recalcitrantes; pudiendo presentarse como Erupción rosácea-símil, Rosácea granulomatosa-símil, Erupción dermatitis perioral-símil o rosácea esteroidal. Para hacer un correcto diagnóstico, los hallazgos clinicopatológicos deben ser concordantes, se debe confirmar con examen con $\mathrm{KOH}$ o la SSSB y debe haber respuesta efectiva a terapia acaricida. El tratamiento de estas condiciones aún no está bien establecido, con evidencia contradictoria respecto de la efectividad de los mismos, existiendo múltiples esquemas según el cuadro clínico y grado de extensión de compromiso, y encontrándose aún poca evidencia de nivel 1 para sustentar eventuales recomendaciones.

\section{REFERENCIAS BIBLIOGRÁFICAS}

1. Ayres S Jr, Ayres S III. Demodectic eruptions (demodicidosis) in the human: 30 years' experience with 2 commonly unrecognized entities; pityriasis folliculorum (Demodex) and acne rosacea (Demodex type). Arch Dermatol 1961; 83:816-27

2. Baima B, Sticherling M. Demodicidosis revisited. Acta Derm Venereol 2002; 82:3-6

3. Forton F, Germaux MA, Brasseur T, De Liever A, Laporte M, Mathys $\mathrm{C}$ et al. Demodicosis and rosacea: Epidemiology and significance in daily dermatologic practice. J Am Acad Dermatol 2005; 52:74-87

4. Hsu C-K, Hsu M, Lee J. Demodicosis: A clinicopathological study. J Am Acad Dermatol 2009; 60:453-62

5. Ozdemir MH, Aksoy U, Sonmez E, Akisu C, Yorulmaz C, Hilal A. Prevalence of Demodex in health personnel working in the autopsy room. Am J Forensic Med Pathol 2005; 26:18-23

6. Elston DM. Demodex mites: Facts and controversies. Clin Dermatol $2010 ; 28: 502-4$

7. Lacey N, Delaney S, Kavanagh K, Powell FC. Mite-related bacterial antigens stimulate inflammatory cells in rosacea. Br J Dermatol 2007; 157:474-81

8. Bonnar E, Eustace P, Powell FC. The Demodex mite population in rosacea. J Am Acad Dermatol 1993; 28:443-8

9. Akilov OE, Mumcuoglu KY. Association between human demodicosis and HLA class I. Clin Exp Dermatol 2003; 28:70-3

10. Mumcuoglu KY, Akilov OE. The role of HLA A2 and Cw2 in the pathogenesis of human demodicosis. Dermatology 2005; 210:109-14

11. Clyti E, Sayavong K, Chanthavisouk K. Demodecidosis in a patient infected by HIV: successful treatment with ivermectin. Ann Dermatol Venereol 2005; 132:459-61

12. Lubbe J, Stucky L, Saurat JH. Rosaceiform dermatitis with follicular Demodex after treatment of facial atopic dermatitis with $1 \%$ pimecrolimus cream. Dermatology 2003; 207:204-5

13. Seyhan ME, Karincaoglu Y, Bayram N, Aycan O, Kuku I. Density of Demodex folliculorum in haematological malignancies. J Int Med Res 2004; 32:411-5

14. Eismann R, Bramsiepe I, Danz B, Wohlrab J, Marsch W, Fiedler E. Abscessing nodular demodicosis- therapy with ivermectin and permethrin. J Eur Acad Dermatol Venereol 2010; 24:79-80

15. Woo YR, Lim JH, Cho DH, Park HJ. Rosacea: Molecular Mechanisms and Management of a Chronic Cutaneous Inflammatory Condition. Int J Mol Sci 2016; 17(9):1562

16. Yamasaki K, Di Nardo A, Bardan A, Murakami M, Ohtake T, Coda A, Dorschner RA, Bonnart C, Descargues P, Hovnanian A, Morhenn VB, Gallo RL. Increased serine protease activity and cathelicidin promotes skin inflammation in rosacea. Nat Med 2007; 13(8):975-80

17. Forton F, Germaux MD, Brasseur T. Demodicosis and rosacea: Epidemiology and significance in daily dermatologic practice. J Am Acad Dermatol 2005; 52(1):74-87 
18. Segal R, Mimouni D, Feuerman H, Pagovitz O, David M Dermoscopy as a diagnostic tool in demodicidosis. Int J Dermatol 2010; 49:1018-23

19. Forton F, Seys B, Marchal JL, Song M. Demodex folliculorum and topical treatment: acaricidal action evaluated by standardized skin surface biopsy. Br J Dermatol 1998; 138:461-6

20. Kanada KN, Nakatsuji T, Gallo RL. Doxycycline indirectly inhibits proteolytic activation of tryptic kallikrein-related peptidases and activation of cathelicidin. J Invest Dermatol 2012; 132(5):1435-42

21. Askin Ü, Seckin D. Comparison of the two techniques for measurement of the density of Demodex folliculorum: standardized skin surface biopsy and direct microscopic examination. Br J Dermatol 2010; 162:1124-6
22. Bikowski JB, Del Rosso JQ. Demodex Dermatitis: A Retrospective Analysis of Clinical Diagnosis and Successful Treatment with Topical Crotamiton. J Clin Aesthet Dermatol 2009; 2(1):20-5

23. Jansen T, Kastner U, Kreuter A, Altmeyer P. Rosacea-like demodicidosis associated with acquired immunodeficiency syndrome. Br J Dermatol 2001; 144:139-42

24. Forstinger $\mathrm{C}$, Kittler $\mathrm{H}$, Binder $\mathrm{M}$. Treatment of rosacea-like demodicosis with oral ivermectin and topical permethrin cream. J Am Acad Dermatol 1999; 41:775-7

25. Karincaoglu Y, Miman O, Kalayci B, Aycan O, Atambay M. A demodicosis case which responded to systemic ivermectin. Eur J Dermatol 2009; 19:189-90 